\title{
The Comparison of Classic Image Resizing Methods
}

\author{
Zijuan Zhang \\ Shaanxi Polytechnic Institute, Xianyang, China \\ bluesky1023@126.com
}

\begin{abstract}
In recent years, with the emergence of different size of the display devices, image is inevitably reduced or enlarged (referred to as resizing) to adapt to different display devices, content-aware image resizing technology is becoming a research hotspot. When resizing the images, this technology not only takes into consideration the constraints of geometry size, and at the same time but also consider the content of the image for different content using a different approach. High degrees of important areas are remained relatively unchanged as far as possible, and less important area are changed a few bigger, so as to achieve the aim of resizing. Image resizing technique is also known as image retargeting. So far, the classic image retargeting technology has the following three kinds: seam carving, SNS, multiple-operator. This article aims to analyze the existing three important resizing technology, points out the scope of above three methods' adaptation, compares the advantages and disadvantages, qualitative conclusions of three ways are given finally.
\end{abstract}

Keywords-Image resizing; Adaptation scope; Disadvantage; Comparison

\section{INTRODUCTION}

Content-aware image resizing is also known as image retargeting, which is put forward by Avidan and Shamir [1] at the 2007 SIGGRAPH. From then on, the technology about image retargeting is more and more attractive towards researchers.

Content-aware image resizing is divided into two steps that is identification of the important area and resizing based on the importance in image. Among them, the first step the importance of image reflects the human eyes in the sensitive degree of different changes in the content area, usually based on image gradient and significant degree's calculation. Among them, the image significance reflects human eye's attractive to the image in different content areas, belongs to the category of the visual perception. Many applications based on visual perception such as image retrieval, image segmentation, target detection, video image compression, images, video quality assessment all have a deep research on image significant degree, therefore, the content-aware image resizing technology literature mainly focus on the second stage about scaling technology.

The purpose of this paper is to compare the existing classical scaling technology, gives a qualitative comparison and analysis. Existing classical scaling techniques will be discussed in the following chapters.

\section{SEAM CARVING}

Seam carving resizing method is proposed by Avidan and Shaimr [1] on the Siggraph 2007, the method is also known as Backward seam carving algorithms. Seam carving way refers to through continuously deleting or inserting connected horizontal or vertical seam through the whole image to realize the image retargeting technique. Among them, the width of seam is a pixel, deleting seam in the image reduces the image, on the contrary, copying seam magnifies image. Processing level (vertical) seam is to change the image height (width). In order to keep the main characteristic of image content, seam carving algorithms deletes or inserts seam with the smallest energy every time. The energy of carving seam refers to the sum of pixels in the seam. In the literature, the content importance is regarded as corresponding pixels' gradient value. As shown in figure 1:

\section{SNS}

At Siggraph Asia in 2008, Wang has proposed a optimized scale-and-stretch (SNS) method for image resizing [2]. As shown in Figure2:

In scaling technique based on image distortion, mainly used to regular rectangular grid, irregular triangle grids and the trapezoid grid. Among them, the triangle and the curved trapezoid grid's advantage is that can be divided grids according to the image content feature points, to achieve the same grid pixels with importance value relatively smooth, but the measure of the grid shape is not convenient as rectangular grid. Simple, often finding the center of gravity position. On the contrary, the image of using rectangular mesh, mesh vertex and image feature points rarely overlap, leading to the subject object nearing the border of the grid is often composed of importance compared to the larger pixel, made by the pixel averaging importance of actual pixels grid importance cannot effectively show the important of degree and influence the optimization effect. Rectangular grids, however, as long as keeping aspect geometric scaling can maintain its shape, the grid shape variable optimization function is relatively simple, intuitive.

\section{Multi-Operator Algorithm}

Multi-operator image scaling method [3] combining seam carving, traditional interpolation and cropping of three operation or two to implement content-aware image resizing. The method focuses on how to determine the sequence and quantity of each operation. The most direct ideas is exhaustive trying all the scaling results under different operating sequence and operation, and then choosing the 
optimal results. If allowing to shrink and enlarge cross, list execution sequence is endless. It is usually that when reducing or enlarging image, every operation is prescribed can only make narrow (enlarge) processing. Under this premise, when the kinds of $m$ operations in one direction change the image size to $w$, the optional for operation sequences are $m^{v}$, still are so big, so multi-operator scaling method would give some additional assumptions to reduce the amount of calculation, there are two common hypothesis: one is to look for regular path [3], according to the fact that influence of operating number is greater than operating sequence, by fixed operation sequence in order to simplify the problem to find the optimal number of operation; Another is to look for mixed path [3], firstly fixing the number of sub operations with $k(k \geq 1)$ every time, so as to determine the length of the sequence $n$, then using dynamic programming method to determine the each type of operation. In addition, when looking for the optimal operation type, regarding the situation that the same number for each operation but a different the sequence order as keeping only the optimal value in order to reduce the amount of calculation.

After determining the order and quantity's method of operation sequence, difficulties of multi-operator methods are how to design a good measurement criterion to measure the resized image similarity with the original image, this also is the main difference between different operation methods.

\section{COMPARISON AND CONCLUSIONS}

It is not yet a more precise evaluation of the performance of image resizing methods, therefore, in this paper, regarding to the characters of the above three kinds of image scaling technology, gives some qualitative analysis. First of all, based on seam carving method and the method based on image deformation in speed significantly faster than multioperator, especially more quickly than based on block matching multi-operation. Next, in effect, each of them has its advantages, each has suitable image type, there is not a kind of method which is universal, and cannot replace each other.

Seam carving's quality comes from the characteristics of its discrete pixel level operation. Since it is the pixel level, the result is fine, because it is a discrete switch operation, the result of color value that is retained from the original image is one of the most close to original one. But also because it is discrete, it is easy to damage the image of the structure of information, to produce artificial noise. Therefore, seam carving is suitable for the images with the smooth transition background region, structural is not strong, as shown in figure 3 girl image's resizing result for girls regional characteristics of reserving is relatively good. In addition, seam carving method can also be used for object's deleting.
Multi-operator is the essence that is considered by the main object of deformation, structure information, producing artificial noise and image content's lost to the influence degree of the overall effect in image, and then compromise. Usually, the more ideal operation of image is done by seam carving, when seams damage image structure information, introduce more artificial noise or excessive deleting one regional background information and affect global image visual effect, gives up seam carving, finally uses uniform resizing. At this point, the continuity of the uniform scaling can keep the relative proportion of each part of the image, also can inhibit the production of artificial noise. But, more than most multi-operation optimization process is to find the optimal result, can only obtain a relatively optimal results. As a result, multi-operation's results often give a person a kind of not ideal, but still feel good. In most cases, the multioperation is a good choice when seam carving's results are relatively poor, as shown in figure 3 , multi-operation results of the goldfish in the shape is better than the seam carving's result.

The image scaling base on the deformation in importance and under the boundary condition's constraints, by optimizing the grid shape variables, the sum of the association, a local grid is given different scaling factors, has the continuity of the interpolation resizing and is able to keep the characteristics of the main object in the image. As shown in figure 3 , the characteristics of the eagle keep the best. But when the grid degenerate into a point or a line, the corresponding excessive compression area also can produce image content discontinuity, as shown in figure 3 goldfish image in the left of the area.

The above three kinds of methods are difficult to keep well linear characteristics of image in any direction, as shown in figure 4 the parrot. For images that have relatively few proportion of homogeneous background information, it is hard to get good results. In addition, in the end the stand or fall of scaling results also largely depends on the important degree of image recognition accuracy.

\section{REFERENCES}

[1] S. Avidan, and A. Shamir, "Seam carving for content-aware image resizing," ACM Transactions on Graphics, vol. 26(3), pp.1-10.

[2] Y.S. Wang, C.L. Tai, O. Sorking and T.Y. Lee, "Optimized scaleand-stretch for image resizing," ACM Transactions on Graphics, vol. 27(5), pp.110-118.

[3] W.M. Dong, N. Zhou and J.C. Paul, "Optimized image resizing using seam carving and scaling," ACM Transactions on Graphics, vol. 28(5), pp.1-10.

[4] Z. Karni, D. Freedman, and C. Gotsman, "Energy-based image deformation,"In Computer Graphics Forum, vol.28(5), pp.1257-1268.

[5] Y. Pritch, E. Kav-Venaki, and S. Peleg, "Shift-map image editing," Proceedings of the Twelfth IEEE ICCV. Florida, USA:IEEE, pp.151158.

[6] C. Renjie, D. Freedman, and Z. Karni, "Content-Aware Image Resizing by Quadratic Programming," Proceedings of the 2010 IEEE CVPR Workshop on NORDIA. Florida, USA:IEEE, pp.1-8. 

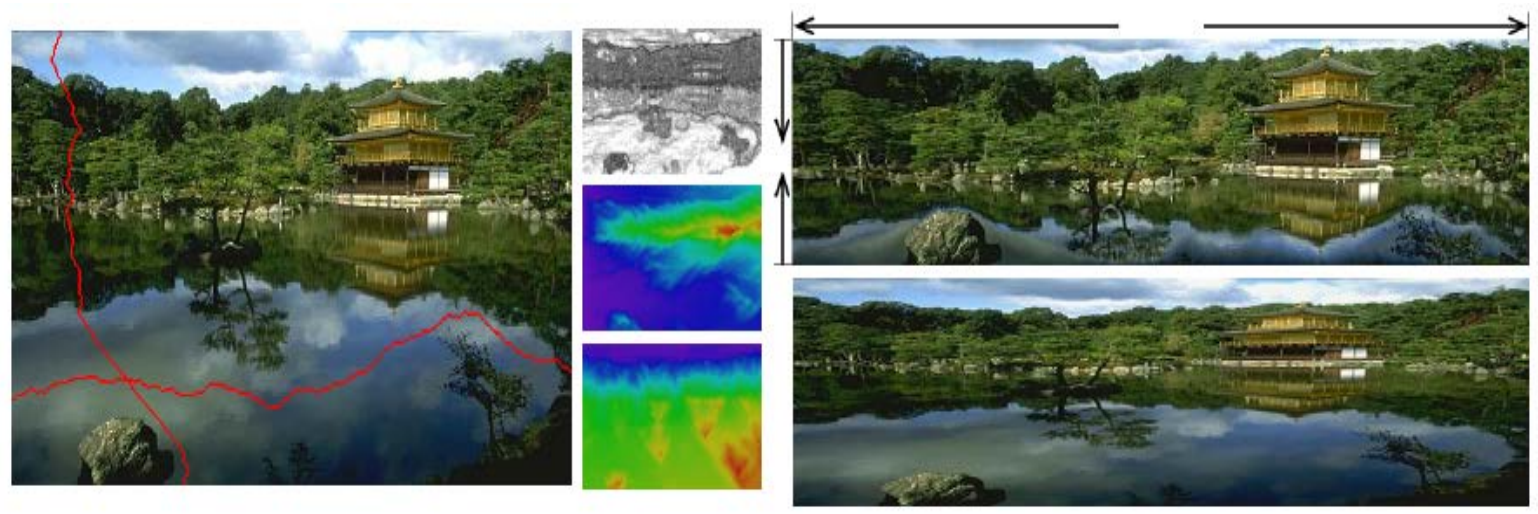

Figure 1. A seam is a connected path of low energy pixels in an image. On the left is the original image with one horizontal and one vertical seam. In the middle the energy function used in this example is shown (the magnitude of the gradient), along with the vertical and horizontal path maps used to calculate the seams. By automatically carving out seams to reduce image size, and inserting seams to extend it, we achieve content-aware resizing. The example on the top right shows our result of extending in one dimension and reducing in the other, compared tostandard scaling on the bottom right. [1]

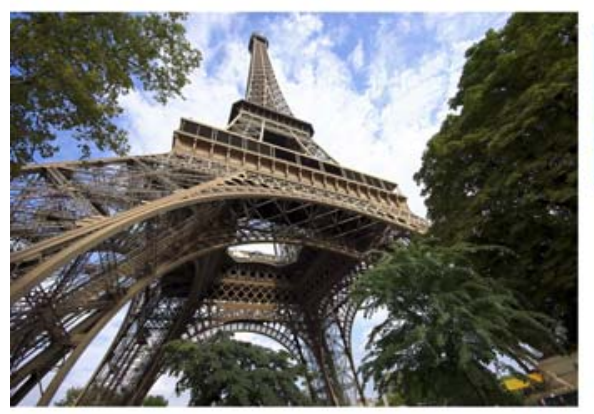

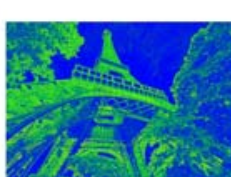

gradient map

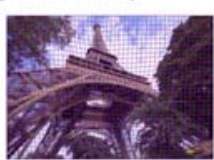

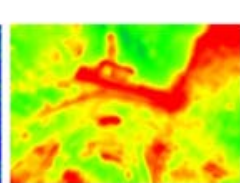

saliency map

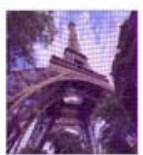

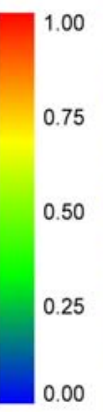

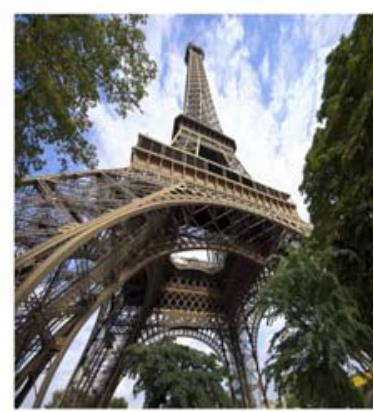

Figure 2. Figure 2: We partition the original image (left) into a grid mesh and deform it to fit the new desired dimensions (right), such that the quad faces covering important image regions are optimized to scale uniformly while regions with homogeneous content are allowed to be distorted. The scaling and stretching of the image content is guided by a significance map which combines the gradient and the saliency maps. (literature [2]) 


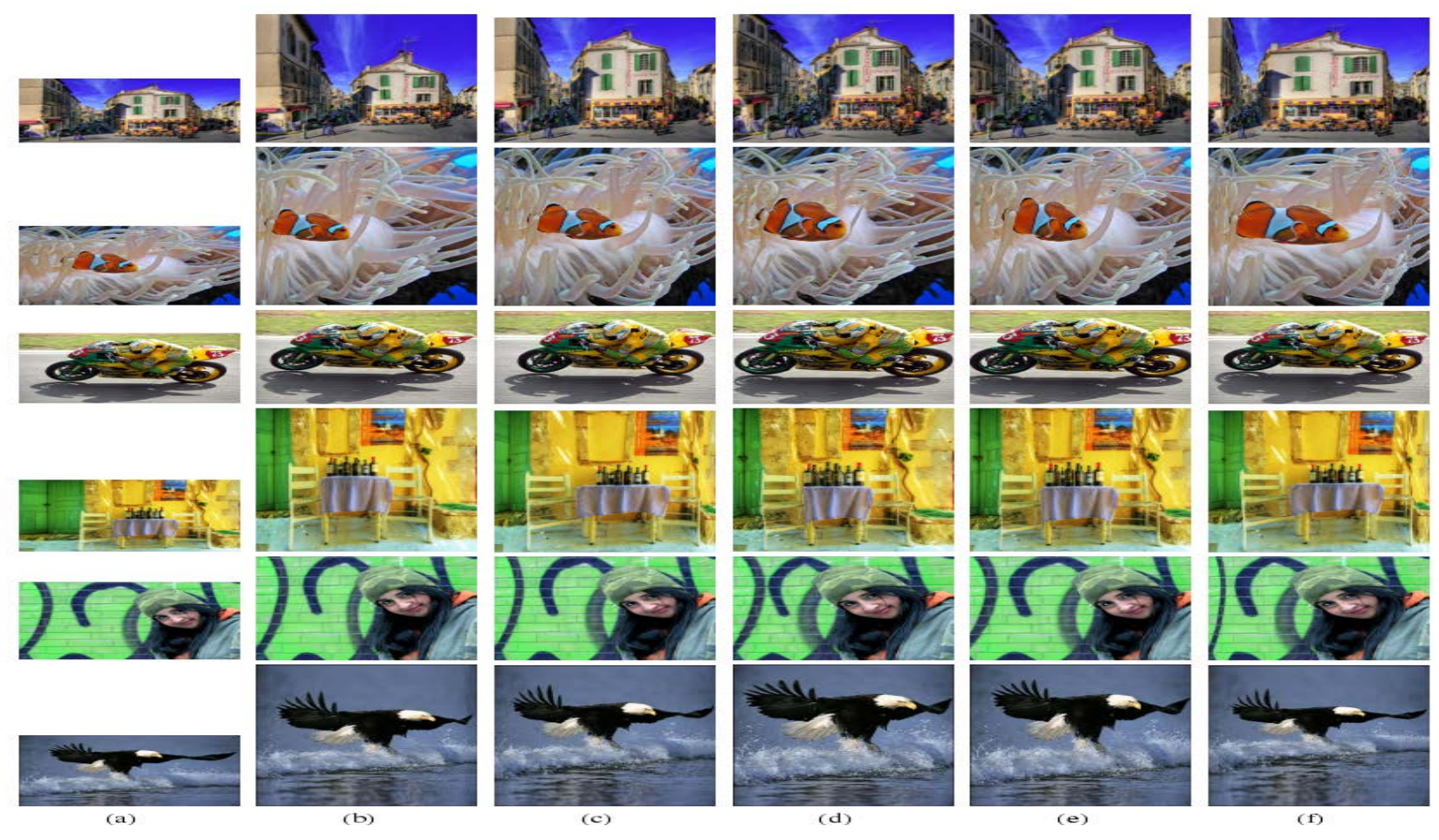

Figure 3. Comparing the proposed resizing method with other competing methods. (a) Original image. (b) Optimized Scale and Stretch(SNS) [2]. (c) Local-Global (LG) [4]. (d) Seam Carving (SC) [1]. (e) Multioperator extension of Seam Carving (MOP) [3]. (f) QP[6]

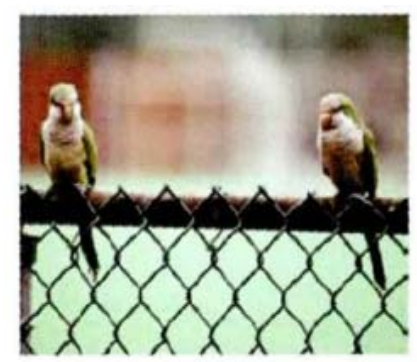

Figure 4.

(a)original image
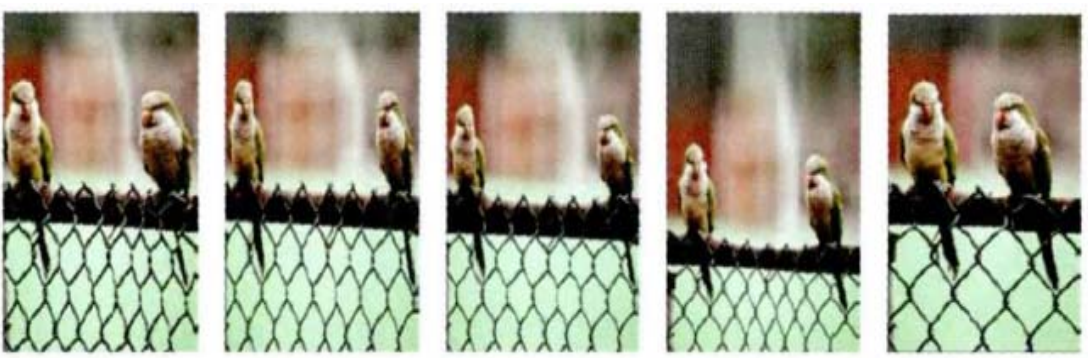

(b)SC[1]

(c)Direct-MOP [3] $\quad$ (d)Indirect-MOP[3]

(e)SNS[2]

(f)Shift-map[5] 